\title{
Aplicação de planejamentos experimentais multivariados à lixiviação de cobre presente em placas de circuito impresso
}

\author{
Rodrigo Ferreira Gomes ${ }^{1}$ \\ Matheus Mello Pereira ${ }^{2}$ \\ Versiane Albis Leão ${ }^{1 *}$
}

\section{Resumo}

O volume de Resíduos de Equipamentos Elétricos e Eletrônicos (REEE) gerados no mundo em 2018 foi de 50 milhões de toneladas. Cerca de 2\% desses resíduos são Placas de Circuito Impresso (PCI) que possuem em sua composição metais nobres e base, sendo o cobre presente em teores de 10-20\%. Além disso, também estão presentes metais tóxicos, tais como chumbo, cádmio e mercúrio, os quais podem acarretar grandes malefícios a natureza e a saúde humana. Portanto, a reciclagem de PCI é uma iniciativa importante, não só devido a questões ambientais, mas também a aspectos econômicos. Diante do exposto, o presente trabalho visou otimizar as variáveis (i) densidade de polpa, (ii) concentração de cloreto férrico hexahidratado, (iii) concentração de ácido clorídrico e (iv) tempo durante a lixiviação do cobre contido em PCI de computadores utilizando planejamentos experimentais multivariados. Os ensaios de lixiviação foram realizados em bateladas em um shaker, sob agitação de $150 \mathrm{~min}^{-1}$ e temperatura de $25 \pm 1^{\circ} \mathrm{C}$. As condições ótimas para a lixiviação de cobre foram densidade de polpa de $120 \mathrm{~g} . \mathrm{L}^{-1}$, concentrações de cloreto férrico hexahidratado e de ácido clorídrico de $1 \mathrm{~mol} . \mathrm{L}^{-1} \mathrm{e}$ tempo de 40 minutos. Para a condição otimizada, a percentagem de lixiviação de cobre foi de $96,22 \pm 1,20 \%$.

Palavras-chave: Placas de Circuito Impresso (PCI); Reciclagem; Hidrometalurgia; Planejamento de experimentos; Metais.

\section{Applying multivariate experimental design to copper leaching from printed circuit boards}

\begin{abstract}
The volume of Waste Electrical and Electronic Equipment (WEEE) produced worldwide in 2018 was 50 million tons. Around $2 \%$ of this waste is Printed Circuit Boards (PCB), which contain noble and base metals, with copper being the most abundant element (10-20\%). Additionally, toxic metals as lead, cadmium and mercury are present, which can cause harmful effects to the environment and human health. Therefore, PCB recycling is an important initiative, not only due to environmental issues but also considering the economic aspects. This research aimed to optimize the variables (i) pulp density, (ii) concentration of ferric chloride hexahydrate, (iii) concentration of hydrochloric acid and (iv) time that influence the copper leaching process contained in PCB of computers through a multivariate experimental design. The leaching assays were carried out in batches, in a shaker, under stirring of $150 \mathrm{~min}^{-1}$ and temperature of $25 \pm 1^{\circ} \mathrm{C}$. The optimum conditions for copper leaching were pulp density of $120 \mathrm{~g} . \mathrm{L}^{-1}$, concentration of ferric chloride hexahydrate and hydrochloric acid of 1mol.L-1 and time of 40 minutes. For the optimized condition, the percentage of copper leaching was $96.22 \pm 1.20 \%$.
\end{abstract}

Keywords: Printed Circuit Boards (PCB); Recycling; Hydrometallurgy; Design of experiments; Metals.

\section{Introdução}

Nos últimos anos, a geração de Resíduos de Equipamentos Elétricos e Eletrônicos (REEE) aumentou em decorrência do aumento do uso destes equipamentos aliado ao crescente ritmo da inovação tecnológica [1]. Além disso, a obsolescência programada se tornou uma estratégia da indústria para diminuir o ciclo de vida dos produtos o que

${ }^{1}$ Laboratório de Bio\&Hidrometalurgia, Departamento de Engenharia Metalúrgica e de Materiais, Universidade Federal de Ouro Preto - UFOP, Ouro Preto, MG, Brasil.

${ }_{2}^{2}$ Programa de Pós-graduação em Engenharia Ambiental - PROAMB, Universidade Federal de Ouro Preto - UFOP, Ouro Preto, MG, Brasil.

*Autor correspondente: versiane@ufop.edu.br

2176-1523 (C) 2020. Gomes et al. Publicado pela ABM. Este é um artigo publicado em acesso aberto (Open Access) sob a licença Creative Commons Attribution, que permite uso, distribuição e reprodução em qualquer meio, sem restrições desde que o trabalho original seja corretamente citado. 
tem provocado um rápido crescimento na taxa de geração de REEE [2-5]. As Placas de Circuito Impresso (PCI) de computadores são um exemplo de REEE e apresentam uma composição diversificada de materiais com potencial de reciclagem, tendo em vista que cerca de $28-30 \%$ representa o conteúdo metálico, sendo esse composto de 10-20\% de cobre e $0,3-0,4 \%$ de metais nobres, tais como ouro, prata, platina, podendo esses apresentar concentrações até dez vezes maiores que em minérios de alto teor. Ademais, também encontram-se presentes $1-5 \%$ de chumbo, 1-3\% de níquel, entre outros [6-8].

As pesquisas sobre métodos apropriados de recuperação de metais presentes nestes recursos são baseadas em princípios pirometalúrgicos, hidrometalúrgicos e biometalúrgicos, bem como suas combinações $[5,9,10]$. Enquanto os processos pirometaúrgicos compreendem a separação térmica dos materiais [11], o tratamento hidrometalúrgico promove a dissolução dos metais em meios ácidos, alcalinos ou salinos a partir do processo de lixiviação seguido por etapas de purificação e separação; uma alternativa interessante, principalmente devido à sua simplicidade, menor gasto energético e menor geração de gases tóxicos se comparado aos processos pirometalúrgicos [12].

A lixiviação de PCI utilizando meio ácido na presença de um agente oxidante é, normalmente, aplicada como o primeiro passo na reciclagem de metais a partir de PCI, após o pré-tratamento mecânico [13-15]. Embora diversos metais sejam lixiviados durante essa etapa, a lixiviação do cobre se torna importante, uma vez que este encontra-se presente em elevadas concentrações (cerca de $20 \%$ em peso) e a lixiviação de outros metais pode ser comprometida pela sua presença, além de acarretar um maior consumo de reagentes. Por esse motivo, sua recuperação é economicamente importante $[13,16]$.

Os processos de lixiviação de cobre a partir de PCI utilizando ácido clorídrico foram estudados por inúmeros autores, sendo que diversos métodos para promover a oxidação de cobre têm sido empregados. Wang e Gaustad [17] utilizaram uma solução de $0,2 \mathrm{~mol} \mathrm{~L}^{-1}$ de peróxido de hidrogênio, 0,8mol. $\mathrm{L}^{-1}$ de ácido clorídrico, densidade de polpa de $4,0 \mathrm{~g} . \mathrm{L}^{-1}$ e temperatura de $25^{\circ} \mathrm{C}$ durante 90 minutos, alcançando uma extração de cobre de $100 \%$. Torres e Lapidus [18] empregaram ar em uma vazão de $1 \mathrm{~L} \cdot \mathrm{min}^{-1}$ para favorecer a oxidação do cobre e utilizaram uma densidade de polpa de $20 \mathrm{~g} . \mathrm{L}^{-1}, 0,5 \mathrm{~mol} . \mathrm{L}^{-1}$ de ácido clorídrico e temperatura de $25^{\circ} \mathrm{C}$, durante um período de 3 horas e obtiveram $85 \%$ de extração de cobre.

Outros meios ácidos também podem ser utilizados para lixiviação de cobre presente em PCI. Lee et al. [19] avaliaram a utilização de ácido nítrico $\left(3,0 \mathrm{~mol}^{\left.-\mathrm{L}^{-1}\right)}\right.$ e ácido sulfúrico $\left(1,2 \mathrm{~mol} . \mathrm{L}^{-1}\right)$ aplicando a seguinte condição experimental: 200g. $\mathrm{L}^{-1}$ de densidade de polpa, temperatura de $25^{\circ} \mathrm{C}$ e tempo de 5 horas. No entanto, utilizando ácido sulfúrico, atingiram valores expressivos de extração de cobre $(75,7 \%)$ apenas na presença de $10 \% \mathrm{~V} \cdot \mathrm{V}^{-1}$ de peróxido de hidrogênio. Por outro lado, obtiveram extração de $96 \%$ de cobre com a utilização de ácido nítrico. O estudo de Caldas [20] também utilizou ácido sulfúrico $\left(2 \mathrm{~mol} . \mathrm{L}^{-1}\right)$ e peróxido de hidrogênio $\left(18,95 \%\right.$ v.v $\left.\mathrm{v}^{-1}\right)$, alcançando $100 \%$ de extração de cobre sob as condições: $53 \mathrm{~g} . \mathrm{L}^{-1}$ de densidade de polpa, temperatura de $95^{\circ} \mathrm{C}$ e tempo de 6 horas.

$\mathrm{O}$ íon férrico $\left(\mathrm{Fe}^{3+}\right)$ é capaz de oxidar a maioria dos metais e está disponível em baixo custo e, por esse motivo apresenta um grande potencial de aplicação como agente oxidante de metais presentes em PCI [21]. Apesar de ter sido pouco empregado na lixiviação de PCI, o íon férrico foi utilizado como agente oxidante em diversos estudos de lixiviação de minerais de cobre. Al-Harahsheh et al. [22] utilizaram cloreto férrico como fonte de $\mathrm{Fe}^{3+}$ e ácido clorídrico para lixiviar calcopirita e obtiveram $20 \%$ de extração de cobre para concentração de ácido clorídrico de $0,5 \mathrm{~mol} . \mathrm{L}^{-1}$, concentração de cloreto férrico de $0,5 \mathrm{~mol} . \mathrm{L}^{-1}$, densidade de polpa de 4 g.L. $\mathrm{L}^{-1}$, temperatura de $90^{\circ} \mathrm{C}$ e tempo de 3 horas. Godočíková et al. [23] extraíram 100\% do cobre contido em um concentrado de sulfeto de $\mathrm{CuPbZn}$ utilizando concentração de ácido clorídrico de $0,2 \mathrm{~mol} . \mathrm{L}^{-1}$, concentração de cloreto férrico de $1,0 \mathrm{~mol} . \mathrm{L}^{-1}$, densidade de polpa de $125 \mathrm{~g} . \mathrm{L}^{-1}$, temperatura de $90^{\circ} \mathrm{C}$ e tempo de 30 minutos. No estudo de Ho et al. [24] também foi alcançada uma extração de $100 \%$ de cobre sob as seguintes condições experimentais: concentração de ácido clorídrico de $0,3 \mathrm{~mol} . \mathrm{L}^{-1}$, concentração de cloreto férrico de 1,5mol.L $\mathrm{L}^{-1}$, densidade de polpa de $50 \mathrm{~g} . \mathrm{L}^{-1}$, temperatura de $90^{\circ} \mathrm{C}$ e tempo de 7 horas.

O uso de ferramentas estatísticas, matemáticas e computacionais vem sendo cada vez mais recorrente, visto que permite a realização de planejamentos experimentais que tem por objetivo fornecer a informação de máxima relevância pela análise de dados [25]. O planejamento de experimentos trata-se de uma abordagem planejada para determinar relações de causa e efeito em que são essenciais: redução ou minimização do número total de ensaios, variação simultânea de todos os fatores e escolha de uma estratégia clara que permita obter soluções confiáveis após cada sequência de experimentos [26,27]. Dentre os diversos tipos de planejamentos experimentais, os sistemas de planejamento fatorial permitem investigar as influências de todas as variáveis experimentais de interesse e os efeitos de interação na resposta ou nas respostas [28].

A Metodologia de Superfície de Resposta (MSR) baseia-se na utilização de um conjunto de técnicas estatísticas e matemáticas que auxiliam na construção de modelos empíricos. Para esse objetivo, empregam-se funções polinomiais lineares ou quadráticas que visam descrever, modelar e otimizar as condições experimentais empregadas no processo ou no sistema de estudo [29]. O planejamento experimental mais empregado na MSR para modelos de segunda ordem é Planejamento Composto Central (CCD), sendo composto por três (3) partes: (i) Um planejamento fatorial completo ou fracionário, ambos nos dois níveis, isto é, cujas variáveis são codificadas como $-1 \mathrm{e}+1$; (ii) Experimentos no ponto central e (iii) Experimentos nos pontos axiais [30]. 
Diante do exposto, o presente trabalho visou otimizar as variáveis densidade de polpa, concentração de cloreto férrico hexahidratado, concentração de ácido clorídrico e tempo durante a lixiviação de cobre contido em PCI de computadores por meio de planejamentos experimentais multivariados.

\section{Materiais e métodos}

As Placas de Circuito Impresso (PCI) foram submetidas à etapa de processamento mecânico, bem como caracterizações física e química em trabalho anterior [31]. Ao final da etapa de separação magnética, obteve-se duas frações: (i) não magnéticas e (ii) magnéticas. Diante dos resultados obtidos nas análises químicas observou-se que o cobre se concentrou preferencialmente na fração não magnética e, por esse motivo, essa foi a fração destinada ao presente trabalho. Essa fração foi composta majoritariamente por cobre $(22,38 \pm 0,16 \%)$, além de outros elementos como cálcio $(5,06 \pm 0,61 \%)$, chumbo $(2,69 \pm 0,88 \%)$, alumínio $(1,25 \pm 0,21 \%)$, ferro $(0,08 \pm 0,01 \%)$, níquel $(0,04 \pm 0,01 \%)$ e zinco $(0,039 \pm 0,007 \%)$ que foram encontrados em menores teores. De acordo com a distribuição granulométrica, observou-se que $90 \%$ das partículas apresentaram granulometria inferior a $1,10 \mathrm{~mm}$. Além disso, $17,12 \%$ das partículas correspondentes à fração não magnética, encontram-se com tamanho inferior a $0,038 \mathrm{~mm}$ (400 mesh).

\subsection{Ensaios de lixiviação}

Para a realização dos experimentos, pesou-se em um béquer cloreto férrico hexahidratado $\left(\mathrm{FeCl}_{3} \cdot 6 \mathrm{H}_{2} \mathrm{O}\right)$ com o auxílio de uma balança analítica da marca Shimadzu ${ }^{\circledR}$, modelo AUY220 e adicionou-se ácido clorídrico $(\mathrm{HCl})$ concentrado, ambos os reagentes da marca Synth ${ }^{\circledR}$. A solução de ácido clorídrico e cloreto férrico hexahidratado foi filtrada em papel de filtro faixa preta, diretamente em um balão volumétrico, aferindo-se o volume com água destilada. Em seguida, pipetou-se $50 \mathrm{~mL}$ da solução em um Erlenmeyer de $250 \mathrm{~mL}$ e verteu-se a amostra de PCI não magnética, após a mesma ser homogeneizada por meio de pilha cônica e pesada. A seguir, o Erlenmeyer foi encaminhado a um shaker termostatizado (New Brunswick Scientific, INNOVA 44), o qual foi operado com agitação de $150 \mathrm{~min}^{-1}$ e mantido a temperatura de $25 \pm 2^{\circ} \mathrm{C}$ pelo tempo correspondente a cada ensaio de lixiviação. Ao final do ensaio, a solução lixiviada foi filtrada em papel de filtro faixa azul, acondicionada em recipientes e encaminhada à análise química em ICP-OES (Varian ${ }^{\circledR}$, modelo 725/ES). Vale ressaltar que a margem de erro inerente esta análise é de $5 \%$.

\subsection{Planejamentos experimentais multivariados}

A partir de informações obtidas na revisão da literatura, por meio dos trabalhos de Wang e Gaustad [17], Torres e Lapidus [18], Lee et al. [19], Caldas [20], Al-Harahsheh et al. [22], Godočíková et al. [23] e Ho et al. [24], optou-se por definir nos planejamentos experimentais multivariados como variável dependente a extração de cobre. As variáveis independentes foram densidade de polpa, concentração de cloreto férrico hexahidratado, concentração de ácido clorídrico e tempo.

A triagem foi a primeira etapa dos planejamentos experimentais multivariados e teve como intuito estimar o efeito dos fatores (variáveis independentes) sobre a resposta (variável dependente). $O$ estudo de triagem foi realizado utilizando-se $o$ software Statistica 13 Academic, a partir de um planejamento fatorial completo $2^{4} \mathrm{com}$ triplicata no ponto central, totalizando 19 ensaios. Os níveis de variação de cada uma das variáveis dependentes estão apresentados na Tabela 1.

De posse dos resultados obtidos na etapa de triagem, foram avaliados os efeitos de cada um dos fatores sobre a resposta (extração de cobre) e definidos os novos níveis a serem aplicados na etapa de Metodologia de Superfície de Resposta (MSR), a qual foi realizada por meio de um planejamento composto central esférico com triplicata no ponto central, totalizando 26 ensaios. Nesta etapa, determinaram-se os valores das variáveis que fornecem a condição ótima de lixiviação, também utilizando-se o software Statistica 13 Academic. As variáveis e os níveis avaliados no planejamento composto central esférico estão apresentados na Tabela 2. Vale ressaltar que o nível de significância adotado em ambos os métodos foi de 0,05 .

\section{Resultados e discussão}

\subsection{Otimização multivariada da extração de cobre}

\subsubsection{Triagem}

A partir da Tabela 3, pode-se observar que nos ensaios $3,7,11$ e 15 foram obtidos os maiores teores de cobre

Tabela 1. Variáveis e níveis estudados no planejamento fatorial completo $2^{4}$ com triplicata no ponto central para lixiviação de cobre. O nível 0 corresponde ao ponto central do planejamento

\begin{tabular}{|c|c|c|c|c|}
\hline \multirow{2}{*}{\multicolumn{2}{|c|}{ Variável (Var.) }} & \multicolumn{3}{|c|}{ Níveis } \\
\hline & & -1 & $\mathbf{0}$ & +1 \\
\hline Var. 1 & Densidade de polpa (g.L - $\left.^{-1}\right)$ & 100,00 & 200,00 & 300,00 \\
\hline Var. 2 & Concentração da solução de $\mathrm{FeCl}_{3} \cdot 6 \mathrm{H}_{2} \mathrm{O}\left(\mathrm{mol} \cdot \mathrm{L}^{-1}\right)$ & 0,10 & 0,55 & 1,00 \\
\hline Var. 3 & Concentração da solução de $\mathrm{HCl}($ mol.L-1-1) & 0,10 & 0,55 & 1,00 \\
\hline Var. 4 & Tempo de lixiviação (minutos) & 45,00 & 142,50 & 240,00 \\
\hline
\end{tabular}


no lixiviado. Todos esses ensaios foram realizados com níveis -1 $\left(100,00 \mathrm{~g} . \mathrm{L}^{-1}\right)$ de densidade de polpa e níveis +1 $\left(1,00 \mathrm{~mol}^{\mathrm{L}} \mathrm{L}^{-1}\right)$ de concentração de cloreto férrico hexahidratado. Em contrapartida, os ensaios 2, 6, 10, 14 foram preparados com níveis $+1\left(300,00 \mathrm{~g} . \mathrm{L}^{-1}\right)$ de densidade de polpa e níveis -1 $\left(0,10 \mathrm{~mol}^{\left.-\mathrm{L}^{-1}\right)}\right.$ de concentração de cloreto férrico hexahidratado, e as extrações de cobre no lixiviado foram insignificantes $(0 \%)$, indicando uma maior dependência da variável resposta com os fatores (i) densidade de polpa e (ii) concentração de cloreto férrico hexahidratado.

Com a finalidade de analisar a qualidade do modelo estatístico empregado na etapa de triagem, realizou-se uma análise de variância (ANOVA) e verificou-se que tanto a regressão do modelo quanto a falta de ajuste foram significativas $(p<0,05)$, indicando que o sistema apesar de robusto, não se ajustou ao modelo matemático linear com curvatura, avaliado pela ANOVA, conforme apresentada na Tabela 4. Por esse motivo, na etapa de otimização foi adotado um modelo matemático quadrático, a fim de se alcançar uma falta de ajuste não significativa. Vale ressaltar que o valor do coeficiente de determinação $\left(\mathrm{R}^{2}\right)$ foi de $99,76 \%$, comprovando que apesar de não se ajustar, o modelo explicou bem os dados experimentais.

A maior dependência do teor de cobre no lixiviado com as variáveis (i) concentração de cloreto férrico hexahidratado e (ii) densidade de polpa foi comprovada por meio do diagrama de Pareto, em que foram as mais significantes, com valores de efeitos padronizados iguais a 159,59 e -107,87, respectivamente. Além disso, vale ressaltar o efeito significativo da interação de segunda ordem entre estas variáveis $(-87,86)$ e da curvatura $(-23,64)$.

Por fim, os valores dos efeitos foram avaliados a partir do teste $t$ de Student, utilizando-se do valor de $p$ correspondente a cada uma das variáveis. De posse dos resultados, verificou-se novamente que os fatores densidade de polpa e concentração de cloreto férrico hexahidratado

Tabela 2. Variáveis e níveis avaliados no planejamento composto central esférico com triplicata no ponto central para lixiviação de cobre

\begin{tabular}{|c|c|c|c|c|c|c|}
\hline \multirow{2}{*}{\multicolumn{2}{|c|}{ Variável (Var.) }} & \multicolumn{5}{|c|}{ Níveis } \\
\hline & & -2 & -1 & $\mathbf{0}$ & +1 & +2 \\
\hline Var. 1 & Densidade de polpa (g.L $\left.\mathrm{L}^{-1}\right)$ & 50,00 & 95,00 & 125,00 & 162,50 & 200,00 \\
\hline Var. 2 & Concentração da solução de $\mathrm{FeCl}_{3} \cdot 6 \mathrm{H}_{2} \mathrm{O}\left(\mathrm{mol} \cdot \mathrm{L}^{-1}\right)$ & 0,50 & 0,75 & 1,00 & 1,125 & 1,50 \\
\hline Var. 3 & Concentração da solução de $\mathrm{HCl}($ mol.L-1 $)$ & 0,25 & 0,50 & 0,75 & 1,00 & 1,25 \\
\hline Var. 4 & Tempo de lixiviação (minutos) & 60,00 & 105,00 & 150,00 & 195,00 & 240,00 \\
\hline
\end{tabular}

Tabela 3. Resultados em termos de percentagem de extração de cobre (p. $\left.\mathrm{p}^{-1}\right)$ do planejamento fatorial completo $2^{4}$ com triplicata no ponto central. Os valores entre parênteses representam os níveis decodificados

\begin{tabular}{|c|c|c|c|c|c|}
\hline Ensaios & $\begin{array}{c}\text { Densidade de polpa } \\
\left(\text { g. } \mathrm{L}^{-1}\right)\end{array}$ & $\begin{array}{c}\text { Concentração de } \\
\mathrm{FeCl}_{3} \cdot 6 \mathrm{H}_{2} \mathrm{O}\left(\mathrm{mol}^{\circ} \mathrm{L}^{-1}\right)\end{array}$ & $\begin{array}{c}\text { Concentração de HCl } \\
\left(\mathrm{mol}^{\left.-L^{-1}\right)}\right.\end{array}$ & Tempo (min) & \% Extração de cobre \\
\hline 1 & $-1(100,00)$ & $-1(0,10)$ & $-1(0,10)$ & $-1(45,00)$ & 9,42 \\
\hline 2 & $1(300,00)$ & $-1(0,10)$ & $-1(0,10)$ & $-1(45,00)$ & 0,18 \\
\hline 3 & $-1(100,00)$ & $1(1,00)$ & $-1(0,10)$ & $-1(45,00)$ & 80,88 \\
\hline 4 & $1(300,00)$ & $1(1,00)$ & $-1(0,10)$ & $-1(45,00)$ & 22,39 \\
\hline 5 & $-1(100,00)$ & $-1(0,10)$ & $1(1,00)$ & $-1(45,00)$ & 1,05 \\
\hline 6 & $1(300,00)$ & $-1(0,10)$ & $1(1,00)$ & $-1(45,00)$ & 0,21 \\
\hline 7 & $-1(100,00)$ & $1(1,00)$ & $1(1,00)$ & $-1(45,00)$ & 98,31 \\
\hline 8 & $1(300,00)$ & $1(1,00)$ & $1(1,00)$ & $-1(45,00)$ & 24,83 \\
\hline 9 & $-1(100,00)$ & $-1(0,10)$ & $-1(0,10)$ & $1(240,00)$ & 10,00 \\
\hline 10 & $1(300,00)$ & $-1(0,10)$ & $-1(0,10)$ & $1(240,00)$ & 0,03 \\
\hline 11 & $-1(100,00)$ & $1(1,00)$ & $-1(0,10)$ & $1(240,00)$ & 99,95 \\
\hline 12 & $1(300,00)$ & $1(1,00)$ & $-1(0,10)$ & $1(240,00)$ & 20,28 \\
\hline 13 & $-1(100,00)$ & $-1(0,10)$ & $1(1,00)$ & $1(240,00)$ & 8,95 \\
\hline 14 & $1(300,00)$ & $-1(0,10)$ & $1(1,00)$ & $1(240,00)$ & 0,03 \\
\hline 15 & $-1(100,00)$ & $1(1,00)$ & $1(1,00)$ & $1(240,00)$ & $104,31 * *$ \\
\hline 16 & $1(300,00)$ & $1(1,00)$ & $1(1,00)$ & $1(240,00)$ & 32,48 \\
\hline $17\left(\mathrm{PC}^{*}\right)$ & $0(200,00)$ & $0(0,55)$ & $0(0,55)$ & $0(142,50)$ & 22,07 \\
\hline $18(\mathrm{PC})$ & $0(200,00)$ & $0(0,55)$ & $0(0,55)$ & $0(142,50)$ & 21,23 \\
\hline $19(\mathrm{PC})$ & $0(200,00)$ & $0(0,55)$ & $0(0,55)$ & $0(142,50)$ & 20,63 \\
\hline
\end{tabular}

*PC: Ponto Central; **O valor superior a $100 \%$ se deve ao erro da análise instrumental, encontrando-se dentro da faixa de erro permitida ( $5 \%)$. Considerar como sendo $100 \%$. 
apresentaram efeitos mais significativos, corroborando com os resultados elucidados pelo diagrama de Pareto. Assim sendo, foram definidos os novos níveis para a etapa subsequente de otimização.

\subsubsection{Metodologia de Superfície de Resposta (MSR)}

Por meio dos resultados obtidos na etapa de otimização, apresentados na Tabela 5, pode-se concluir que as maiores extrações de cobre foram obtidas nos ensaios 6,7 e 8 , alcançando $100 \%$. Como era esperado, em todos esses ensaios, os níveis de densidade de polpa e concentração de cloreto férrico hexahidratado foram respectivamente, -1 $\left(87,50 \mathrm{~g} . \mathrm{L}^{-1}\right)$ e $+1\left(1,25 \mathrm{~mol}^{\mathrm{L}} \mathrm{L}^{-1}\right)$. Além disso, pode-se observar que os níveis adotados na etapa de otimização foram satisfatórios, uma vez que aproximadamente $75 \%$ dos ensaios realizados apresentaram valores de extração superiores a $80 \%$.

A partir da análise de variância (ANOVA), apresentada na Tabela 6 , pode-se avaliar a qualidade do modelo utilizado

Tabela 4. Tabela ANOVA para o modelo matemático linear com curvatura obtido pelo planejamento fatorial completo $2^{4}$ com triplicata no ponto central

\begin{tabular}{cccccc}
\hline FV & SQ & nGL & MQ & F $_{\text {calc. }}$ & p \\
\hline Regressão & $23.700,72$ & 15 & $1.580,05$ & 81,70 & $\mathbf{0 , 0 0 1 9 4}$ \\
Resíduos & 58,014 & 3 & 19,34 & & \multirow{2}{*}{$\mathbf{0 , 0 0 9 0 8}$} \\
Falta de ajuste & 56,97 & 1 & 56,97 & 0,64 \\
Erro puro & 1,05 & 2 & & \\
\hline SQ Total & $23.758,73$ & 18 &
\end{tabular}

Coeficiente de Determinação $\left(\mathrm{R}^{2}\right)=99,76 \%$

FV: fonte de variação; SQ: soma quadrática; nGL: número de graus de liberdade; MQ: média quadrática; $\mathrm{F}_{\text {calc }}$ : valor do teste $\mathrm{F}$ calculado e p: parâmetro estatístico $p$.

Tabela 5. Resultados em termos de percentagem de extração de cobre ( $\left.\mathrm{p} \cdot \mathrm{p}^{-1}\right)$ do planejamento composto central esférico com triplicata no ponto central. Os valores entre parênteses representam os níveis decodificados

\begin{tabular}{|c|c|c|c|c|c|}
\hline Ensaios & $\begin{array}{c}\text { Densidade de polpa } \\
\left(\text { g. } \mathrm{L}^{-1}\right)\end{array}$ & $\begin{array}{c}\text { Concentração de } \\
\mathrm{FeCl}_{3} \cdot 6 \mathrm{H}_{2} \mathrm{O}\left(\mathrm{mol}^{-\mathrm{L}^{-1}}\right)\end{array}$ & $\begin{array}{c}\text { Concentração de HCl } \\
\left(\mathrm{mol}^{\left.-L^{-1}\right)}\right.\end{array}$ & Tempo (min) & \% Extração de cobre \\
\hline 1 & $-1(87,50)$ & $-1(0,75)$ & $-1(0,50)$ & $-1(105,00)$ & 83,62 \\
\hline 2 & $-1(87,50)$ & $-1(0,75)$ & $-1(0,50)$ & $1(195,00)$ & 96,33 \\
\hline 3 & $-1(87,50)$ & $-1(0,75)$ & $1(1,00)$ & $-1(105,00)$ & 92,77 \\
\hline 4 & $-1(87,50)$ & $-1(0,75)$ & $1(1,00)$ & $1(195,00)$ & 98,56 \\
\hline 5 & $-1(87,50)$ & $1(1,25)$ & $-1(0,50)$ & $-1(105,00)$ & 92,12 \\
\hline 6 & $-1(87,50)$ & $1(1,25)$ & $-1(0,50)$ & $1(195,00)$ & $102,22 * *$ \\
\hline 7 & $-1(87,50)$ & $1(1,25)$ & $1(1,00)$ & $-1(105,00)$ & $100,66^{* *}$ \\
\hline 8 & $-1(87,50)$ & $1(1,25)$ & $1(1,00)$ & $1(195,00)$ & $101,31 * *$ \\
\hline 9 & $1(162,50)$ & $-1(0,75)$ & $-1(0,50)$ & $-1(105,00)$ & 46,28 \\
\hline 10 & $1(162,50)$ & $-1(0,75)$ & $-1(0,50)$ & $1(195,00)$ & 46,75 \\
\hline 11 & $1(162,50)$ & $-1(0,75)$ & $1(1,00)$ & $-1(105,00)$ & 51,49 \\
\hline 12 & $1(162,50)$ & $-1(0,75)$ & $1(1,00)$ & $1(195,00)$ & 53,63 \\
\hline 13 & $1(162,50)$ & $1(1,25)$ & $-1(0,50)$ & $-1(105,00)$ & 86,21 \\
\hline 14 & $1(162,50)$ & $1(1,25)$ & $-1(0,50)$ & $1(195,00)$ & 91,42 \\
\hline 15 & $1(162,50)$ & $1(1,25)$ & $1(1,00)$ & $-1(105,00)$ & 88,52 \\
\hline 16 & $1(162,50)$ & $1(1,25)$ & $1(1,00)$ & $1(195,00)$ & 98,39 \\
\hline 17 & $-2(50,00)$ & $0(1,00)$ & $0(0,75)$ & $0(150,00)$ & 97,70 \\
\hline 18 & $2(200,00)$ & $0(1,00)$ & $0(0,75)$ & $0(150,00)$ & 53,06 \\
\hline 19 & $0(125,00)$ & $-2(0,50)$ & $0(0,75)$ & $0(150,00)$ & 36,45 \\
\hline 20 & $0(125,00)$ & $2(1,50)$ & $0(0,75)$ & $0(150,00)$ & 92,90 \\
\hline 21 & $0(125,00)$ & $0(1,00)$ & $-2(0,25)$ & $0(150,00)$ & 76,20 \\
\hline 22 & $0(125,00)$ & $0(1,00)$ & $2(1,25)$ & $0(150,00)$ & 88,39 \\
\hline 23 & $0(125,00)$ & $0(1,00)$ & $0(0,75)$ & $-2(60,00)$ & 84,27 \\
\hline 24 & $0(125,00)$ & $0(1,00)$ & $0(0,75)$ & $2(240,00)$ & 97,71 \\
\hline $25\left(\mathrm{PC}^{*}\right)$ & $0(125,00)$ & $0(1,00)$ & $0(0,75)$ & $0(150,00)$ & 86,87 \\
\hline $26(\mathrm{PC})$ & $0(125,00)$ & $0(1,00)$ & $0(0,75)$ & $0(150,00)$ & 89,29 \\
\hline $27(\mathrm{PC})$ & $0(125,00)$ & $0(1,00)$ & $0(0,75)$ & $0(150,00)$ & 86,19 \\
\hline
\end{tabular}

*PC: Ponto Central; **Os valores superiores a $100 \%$ se devem ao erro da análise instrumental, encontrando-se dentro da faixa de erro permitida (5\%). Considerar como sendo $100 \%$. 
na etapa de otimização. A regressão do modelo foi considerada significativa, apresentando $p=1,07557 \times 10^{-7}(p<0,05)$ e o valor do coeficiente de regressão $\left(\mathrm{R}^{2}\right)$ foi de $97,75 \%$, o que comprova que o modelo é capaz de explicar bem os dados. $\mathrm{O}$ valor do teste $F$ para a falta de ajuste indica que esta não teve significância estatística, pois apresentou $F_{\text {calc. }}(8,457)$ cerca de duas vezes inferior ao valor do $F_{\text {tab. }}(19,40)$, calculado de acordo com Skoog et al. [32]. Além disso, observa-se que a falta de ajuste apresentou $(p=0,110303)$ valor superior ao nível de significância adotado $(0,05)$ e por isso indicando a significância estatística do modelo adotado.

Diante do exposto, constatou-se que o modelo adotado na etapa de otimização apresentou boa significância estatística. Por esse motivo, foram calculados os valores dos efeitos e dos coeficientes de regressão, com seus respectivos valores de $p$. Os efeitos mais significativos foram para as variáveis lineares concentração de cloreto férrico hexahidratado $(25,3600)$, densidade de polpa $(-24,5150)$ e da interação entre elas $(17,6700)$. Além disso, as variáveis concentração de ácido clorídrico (linear), tempo (linear) e tempo (quadrática) também revelaram efeitos significativos, sendo estes, respectivamente 5,3967, 6,1517 e 3,3725. Os coeficientes de regressão em termos das variáveis originarias também foram calculados e empregados na determinação do modelo quadrático que descreve o sistema estudado no presente trabalho (Equação 1).

$$
\begin{aligned}
& \hat{y}=74,2825-16,4305 x_{1}-0,6297 x_{1}^{2}+ \\
& 35,6433 x_{3}-7,7413 x_{3}^{2}-0,1109 x_{4}+0,0008 x_{4}^{2}+ \\
& 18,8946 x_{1} x_{2}+0,2680 x_{1} x_{3}-0,0088 x_{1} x_{4}- \\
& 6,9148 x_{2} x_{3}+0,0242 x_{2} x_{4}-0,0537 x_{3} x_{4}
\end{aligned}
$$

A variável dependente $(\hat{y})$ representa o valor predito para a extração de cobre e as variáveis independentes $\left(x_{1}\right.$. $\left.x_{2 ;} x_{3 ;} x_{4}\right)$ os valores de densidade de polpa, concentração de cloreto férrico hexahidratado, concentração de ácido clorídrico e tempo, respectivamente.

Com a finalidade de verificar o ajuste dos dados obtidos experimentalmente com os calculados pela Equação 1, utilizaram-se os valores observados e preditos para calcular os resíduos correspondentes a cada um dos ensaios. O maior valor de resíduo apreciado foi de 6,074 e a média quadrática de todos os resíduos apresentou valor de 2,441.
Por fim, foram obtidas as superfícies de resposta, as quais indicaram o valor esperado para a extração de cobre em função de duas das variáveis independentes estudadas. Cumpre salientar que as demais variáveis não apresentadas em cada uma das superfícies tiveram seus valores fixados nos respectivos pontos centrais, ou seja, densidade de polpa $(125,00)$, concentração de cloreto férrico hexahidratado $(1,00)$, concentração de ácido clorídrico $(0,75)$ e tempo $(150,00)$.

A partir da análise das curvas apresentadas na Figura 1, pode-se observar que em (A), (C), (D) e (E) os valores de extração de cobre são ditados pelas variáveis densidade de polpa e concentração de cloreto férrico hexahidratado, uma vez que as superfícies evidenciaram variações na extração para as variáveis (i) tempo e (ii) concentração de ácido clorídrico próximas a linearidade. Além disso, a partir da superfície (B) observa-se que para uma percentagem de extração de cobre de aproximadamente $100 \%$, os valores de densidade de polpa podem variar de $40 \mathrm{~g} . \mathrm{L}^{-1}$ a $120 \mathrm{~g} . \mathrm{L}^{-1}$, desde que a concentração de cloreto férrico hexahidratado também seja alterada para valores em um intervalo entre $0,6 \mathrm{~mol} . \mathrm{L}^{-1}$ e $1,4 \mathrm{~mol} \cdot \mathrm{L}^{-1}$. A curva representada por $(\mathrm{F})$ indica que as maiores extrações de cobre situam-se em regiões cuja concentração de ácido clorídrico é superior a $0,8 \mathrm{~mol} \cdot \mathrm{L}^{-1}$. No entanto, ao se observar a escala na superfície de resposta, consta-se que em quase toda a superfície a extração de cobre é superior a $80 \%$.

Com o objetivo de examinar os resultados obtidos visando possíveis aplicações industriais, foram selecionados níveis para as variáveis dependentes onde as condições de lixiviação fossem as melhores possíveis, ou seja, baixo custo, alta eficiência e menor tempo. O tempo de lixiviação escolhido foi de 40 minutos, o menor possível visando uma extração de, aproximadamente, $100 \%$ de cobre. Em se tratando da densidade de polpa, o valor fixado foi de $120 \mathrm{~g} . \mathrm{L}^{-1}$, levando-se em consideração a grande correlação deste fator com variável resposta e a aplicação da maior quantidade possível de sólidos, associada a uma elevada eficiência de lixiviação. As concentrações dos reagentes (ácido clorídrico e cloreto férrico hexahidratado) foram ambas definidas como sendo $1 \mathrm{~mol} . \mathrm{L}^{-1}$. Porém, para concentrações de cloreto férrico hexahidratado inferiores a 0,8mol.L $\mathrm{L}^{-1}$ as porcentagens de extração de cobre são baixas, devido à grande dependência destas variáveis. O mesmo não é observado para a concentração de ácido clorídrico, de maneira que esta pode ser inferior a $0,2 \mathrm{~mol} . \mathrm{L}^{-1}$, mantendo-se extrações significativas de cobre.

Tabela 6. Tabela ANOVA para o modelo matemático quadrático obtida pelo planejamento composto central esférico com triplicata no ponto central

\begin{tabular}{cccccc}
\hline FV & SQ & nGL & MQ & $\mathbf{F}_{\text {calc. }}$ & p \\
\hline Regressão & $9.969,95$ & 14 & 712,139 & 37,185 & $\mathbf{1 , 0 7 5 5 7 \times 1 0}^{-7}$ \\
Resíduos & 229,82 & 12 & 19,151 & 8,457 & 0,110303 \\
Falta de ajuste & 224,51 & 10 & 22,451 & 2,655 & \\
Erro puro & 5,31 & 2 & & \\
SQ Total & $10.199,76$ & 26 & \\
& \multicolumn{2}{c}{ Coeficiente de Determinação $\left(\mathrm{R}^{2}\right)=97,75 \%$} & \\
\hline
\end{tabular}

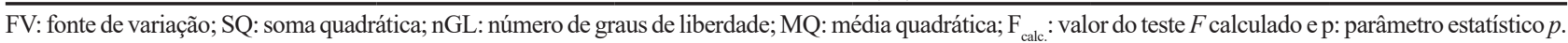


Cumpre salientar que as condições ótimas preditas pelo software Statistica 13 Academic foram distintas das citadas anteriormente, visto que estas são calculadas por meio dos pontos críticos da Equação 1 e não levam em consideração aspectos econômicos e industriais. Para fins comparativos, ambas as condições ótimas para lixiviação de cobre presente em PCI, encontram-se descritas na Tabela 7. As percentagens de extração de cobre para os pontos críticos e para a condição
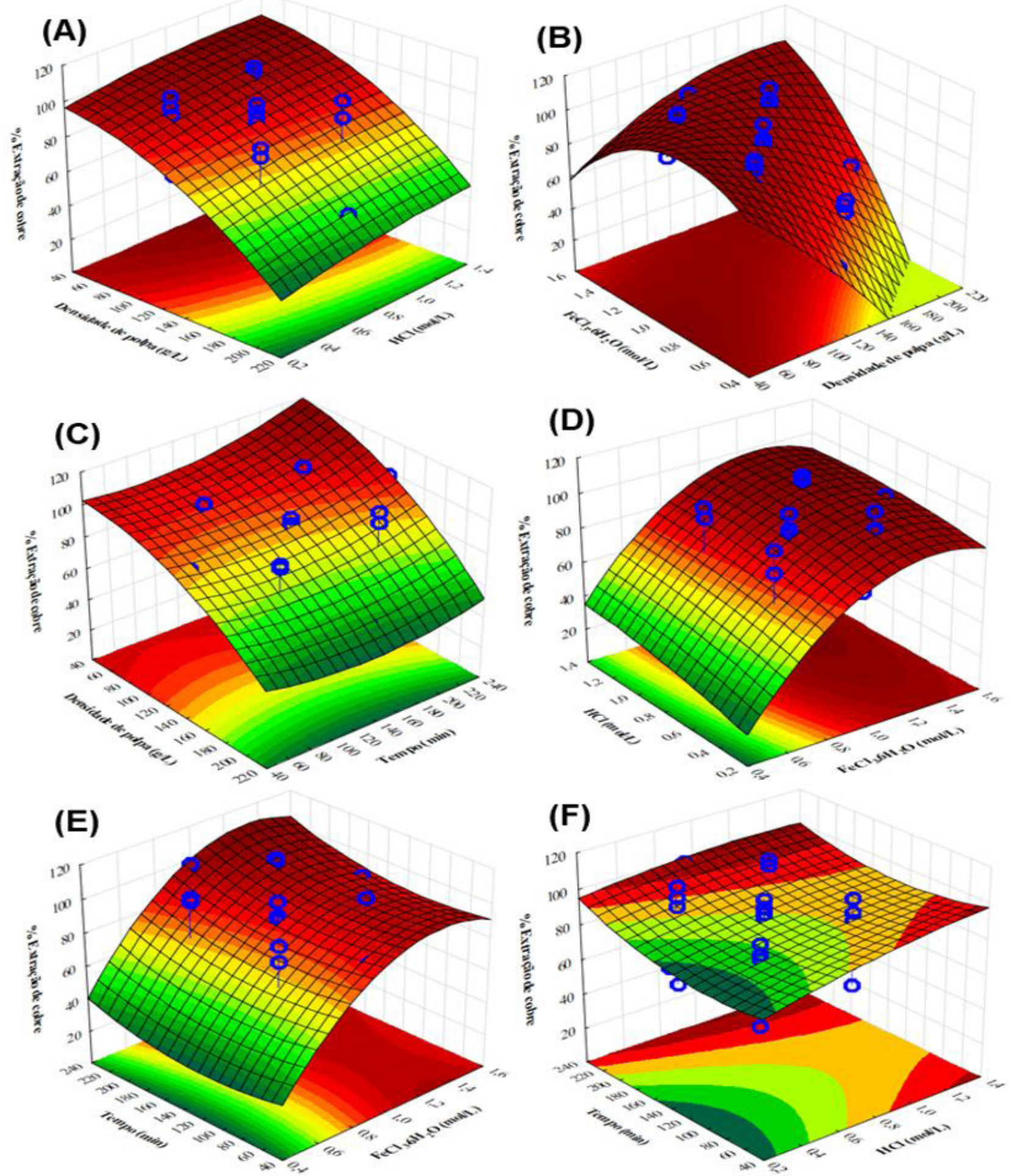

Figura 1. (A), (B), (C), (D), (E) e (F) superfícies de resposta obtidas para a variável extração de cobre (\% p.p $\left.\mathrm{p}^{-1}\right)$. (A) variáveis densidade de polpa* $\mathrm{HCl}$; (B) variáveis $\mathrm{FeCl}_{3} \cdot 6 \mathrm{H}_{2} \mathrm{O} *$ densidade de polpa; (C) variáveis densidade de polpa*Tempo; (D): variáveis $\mathrm{FeCl}_{3} \cdot 6 \mathrm{H}_{2} \mathrm{O} * \mathrm{HCl}$; $(\mathrm{E})$ variáveis $\mathrm{FeCl}_{3} .6 \mathrm{H}_{2} \mathrm{O} *$ Tempo; e (F) variáveis Tempo*HCl. 
Tabela 7. Ponto crítico e condição ótima para a extração de cobre contido nas placas de circuito impresso não magnéticas

\begin{tabular}{lccccc}
\hline & Variável & $\begin{array}{c}\text { Mínimo } \\
\text { Observado }\end{array}$ & $\begin{array}{c}\text { Ponto crítico da } \\
\text { equação }\end{array}$ & $\begin{array}{c}\text { Condição ótima } \\
\text { Observado }\end{array}$ \\
\hline Var. 1 & Densidade de polpa $\left(\mathrm{g} \cdot \mathrm{L}^{-1}\right)$ & 50,000 & 137,076 & 120,000 & 200,000 \\
Var. 2 & Concentração da solução de $\mathrm{FeCl} \cdot 6 \mathrm{H}_{2} \mathrm{O}\left(\mathrm{mol}_{\mathrm{L}} \mathrm{L}^{-1}\right)$ & 0,500 & 1,386 & 1,000 & 1,500 \\
Var. 3 & Concentração da solução de $\mathrm{HCl}\left(\mathrm{mol} \cdot \mathrm{L}^{-1}\right)$ & 0,250 & $\mathbf{1 , 3 8 1 *}$ & 1,000 & 1,250 \\
Var. 4 & Tempo de lixiviação (minutos) & 60,000 & 127,417 & $\mathbf{4 0 , 0 0 0 *}$ & 240,000 \\
\hline
\end{tabular}

*Valores extrapolados (maior que o máximo observado ou menor que o mínimo observado).

ótima definidas foram de 97,33\% e 95,02\%, respectivamente. Estas condições experimentais foram reproduzidas, buscando a validação do modelo. Após a realização dos ensaios sob tais condições foram obtidos os teores médios de extração de cobre de $98,19 \pm 0,94 \%$ e $96,22 \pm 1,20 \%$.

Os estudos realizados por Al-Harahsheh et al. [22], Godočíková et al. [23] e Ho et al. [24] também utilizaram o par cloreto férrico e ácido clorídrico para promover a lixiviação de cobre, porém com o objetivo de lixiviar minérios. Apesar disto, salienta-se a proximidade das concentrações de ácido clorídrico e cloreto férrico utilizadas, uma vez que essas encontraram-se entre $0,3 \mathrm{~mol} . \mathrm{L}^{-1}$ e $0,5 \mathrm{~mol} \cdot \mathrm{L}^{-1}$ e $0,5 \mathrm{~mol} . \mathrm{L}^{-1}$ e $1,5 \mathrm{~mol} . \mathrm{L}^{-1}$, respectivamente, semelhantes às empregadas no presente trabalho. A respeito dos estudos realizados com PCI, Havlik et al. [33], Torres e Lapidus [18] e Wang e Gaustad [17] utilizaram ácido clorídrico em seus estudos, entretanto não empregaram cloreto férrico para que fosse promovida a oxidação do cobre. Ao invés disso, lançaram mão de outros métodos, tais como: utilização de peroxido de hidrogênio, aplicação de grandes tempos de lixiviação (24 horas), aspersão de ar e utilização de baixas densidades de polpas $\left(7,5 \mathrm{~g} \cdot \mathrm{L}^{-1}\right)$. Destaca-se também a utilização de tempos de lixiviação superiores ao encontrado no presente estudo (40 minutos) e densidades de polpa inferiores a $120 \mathrm{~g} . \mathrm{L}^{-1}$ em todos esses trabalhos.

\section{Conclusões}

No presente estudo, utilizou-se um planejamento fatorial completo $2^{4}$ com ponto central e Metodologia de
Superfície de Resposta (MSR) a partir de um planejamento composto central esférico para determinar os efeitos das variáveis densidade de polpa, concentração de cloreto férrico hexahidratado, concentração de ácido clorídrico e tempo na lixiviação de cobre presente em PCI de computadores. De posse dos resultados, verificou-se que os fatores densidade de polpa e concentração de cloreto férrico hexahidratado apresentaram os efeitos mais significativos e, portanto, pode-se inferir que o modelo estatístico proposto conseguiu descrever o comportamento físico esperado para o sistema. Para uma percentagem de extração de cobre de, aproximadamente $100 \%$, os valores de densidade de polpa podem variar de $40 \mathrm{~g} . \mathrm{L}^{-1}$ a $120 \mathrm{~g} . \mathrm{L}^{-1}$, desde que a concentração de cloreto férrico hexahidratado também seja alterada para valores compreendidos em um intervalo de $0,6 \mathrm{~mol}^{-\mathrm{L}^{-1}} \mathrm{a}$ $1,4 \mathrm{~mol} \cdot \mathrm{L}^{-1}$. Os valores ótimos para os parâmetros estudados foram definidos como sendo concentração de cloreto férrico hexahidratado e ácido clorídrico de $1 \mathrm{~mol}^{\mathrm{L}} \mathrm{L}^{-1}$, densidade de polpa de $120 \mathrm{~g} . \mathrm{L}^{-1}$ e tempo de lixiviação de 40 minutos. $\mathrm{Na}$ condição otimizada, a percentagem de lixiviação de cobre foi de $96,22 \pm 1,20 \%$.

\section{Agradecimentos}

Os autores agradecem ao Núcleo de Tecnologia da Informação (NTI) da Universidade Federal de Ouro Preto (UFOP) pelo fornecimento das placas de circuito impresso de computadores obsoletos, à CAPES pela concessão da bolsa de mestrado para um dos autores, à UFMG, bem como ao CNPq, a Finep e a Fapemig.

\section{Referências}

1 Chu Y, Chen M, Chen S, Wang B, Fu K, Chen H. Micro-copper powders recovered from waste printed circuit boards by electrolysis. Hydrometallurgy. 2015;156:152-157. http://dx.doi.org/10.1016/j.hydromet.2015.06.006.

2 Beatriz M. Obsolescência programada e teoria do decrescimento versus direito ao desenvolvimento e ao consumo (Sustentáveis). Veredas do Direito. 2012;9(17):181-196.

3 Henrique S Jr, Moura F, Correa R, Afonso J, Vianna C, Mantovano J. Processamento de placas de circuito impresso de equipamentos eletroeletrônicos de pequeno porte. Química Nova. 2013;36(4):570-576. http://dx.doi.org/10.1590/ S0100-40422013000400015.

4 Estrada-Ruiz R, Flores-Campos R, Gámez-Altamirano H, Velarde-Sánchez E. Separation of the metallic and non-metallic fraction from printed circuit boards employing green technology. Journal of Hazardous Materials. 2016;311:91-99. http://dx.doi.org/10.1016/j.jhazmat.2016.02.061. 
5 Neto I, Sousa C, Brito M, Futuro A, Soares H. A simple and nearly-closed cycle process for recycling copper with high purity from end life printed circuit boards. Separation and Purification Technology. 2016;164:19-27. http:// dx.doi.org/10.1016/j.seppur.2016.03.007.

6 Sarvar M, Salarirad M, Shabani M. Characterization and mechanical separation of metals from computer Printed Circuit Boards (PCBs) based on mineral processing methods. Waste Management. 2015;45:246-257. http://dx.doi. org/10.1016/j.wasman.2015.06.020.

7 Betts K. Reducing the global impact of e-waste. Environmental Science \& Technology. 2008;42(5):1393. http:// dx.doi.org/10.1021/es087087p.

8 Yamane L, Moraes V, Espinosa D, Tenório J. Recycling of WEEE: characterization of spent printed circuit boards from mobile phones and computers. Waste Management. 2011;31(12):2553-2558. http://dx.doi.org/10.1016/j. wasman.2011.07.006.

9 Tesfaye F, Lindberg D, Hamuyuni J, Taskinen P, Hupa L. Improving urban mining practices for optimal recovery of resources from e-waste. Minerals Engineering. 2017;111:209-221. http://dx.doi.org/10.1016/j.mineng.2017.06.018.

10 Kumar A, Holuszko M, Espinosa D. E-waste: An overview on generation, collection, legislation and recycling practices. Resources, Conservation and Recycling. 2017;122:32-42. http://dx.doi.org/10.1016/j. resconrec.2017.01.018.

11 Yamane L. Recuperação de metais de placas de circuito impresso de computadores obsoletos através de processo biohidrometalúrgico [tese]. São Paulo: Escola Politécnica, Universidade de São Paulo; 2012. http://dx.doi. org/10.11606/T.3.2012.tde-07062013-154359.

12 Chen M, Huang J, Ogunseitan O, Zhu N, Wang Y. Comparative study on copper leaching from waste printed circuit boards by typical ionic liquid acids. Waste Management. 2015;41:142-147. http://dx.doi.org/10.1016/j. wasman.2015.03.037.

13 Birloaga I, De Michelis I, Ferella F, Buzatu M, Vegliò F. Study on the influence of various factors in the hydrometallurgical processing of waste printed circuit boards for copper and gold recovery. Waste Management. 2013;33(4):935-941. http://dx.doi.org/10.1016/j.wasman.2013.01.003.

14 Yang H, Liu J, Yang J. Leaching copper from shredded particles of waste printed circuit boards. Journal of Hazardous Materials. 2011;187(1-3):393-400. http://dx.doi.org/10.1016/j.jhazmat.2011.01.051.

15 Souza C. Copper extraction from electronic scraps by oxidative acid leaching process printed circuit board coming from several types of equipments. In: Centre for Mineral Technology. Proceedings of the 6th International Seminar on Copper Hydrometallurgy; 2011 July 6-8; Viña del Mar, Chile. Brazil: CETEM; 2011. p. 1-8.

16 Oh C, Lee S, Yang H, Ha T, Kim M. Selective leaching of valuable metals from waste printed circuit boards. Journal of the Air \& Waste Management Association. 2003;53(7):897-902. http://dx.doi.org/10.1080/10473289 .2003 .10466230 .

17 Wang X, Gaustad G. Prioritizing material recovery for end-of-life printed circuit boards. Waste Management. 2016;31:917-924. https://doi.org/10.1016/j.wasman.2012.05.005.

18 Torres R, Lapidus G. Copper leaching from electronic waste for the improvement of gold recycling. Waste Management. 2016;57:131-139. http://dx.doi.org/10.1016/j.wasman.2016.03.010.

19 Lee J, Kumar M, Kim M-S, Jeong J, Yoo K. Leaching of metals from waste printed circuit boards (WPCBs) using sulfuric and nitric acids. Environmental Engineering and Management Journal. 2014;13(10):2601-2607. http:// dx.doi.org/10.30638/eemj.2014.290.

20 Caldas M. Síntese de nanopartículas de prata a partir de reciclagem de clacas de circuito impresso [tese]. São Paulo: Escola Politécnica, Universidade de São Paulo; 2017.

21 Deveci H, Ball T. A visual insight into the oxidation of sulfide minerals during bioleaching and chemical leaching of a complex ore. Mineral Processing and Extractive Metallurgy Review. 2010;31(3):176-190. http://dx.doi.org/10.108 $0 / 08827508.2010 .482859$.

22 Al-Harahsheh M, Kingman S, Al-Harahsheh A. Ferric chloride leaching of chalcopyrite: synergetic effect of $\mathrm{CuCl}_{2}$. Hydrometallurgy. 2008;91(1-4):89-97. http://dx.doi.org/10.1016/j.hydromet.2007.11.011.

23 Godočíková E, Baláž P, Boldižárová E. Structural and temperature sensitivity of the chloride leaching of copper, lead and zinc from a mechanically activated complex sulphide. Hydrometallurgy. 2002;65(1):83-93. http://dx.doi. org/10.1016/S0304-386X(02)00094-4. 
24 Ho K, Mohapatra D, Reddy B. A study on the acidified ferric chloride leaching of a complex $(\mathrm{Cu}-\mathrm{Ni}-\mathrm{Co}-\mathrm{Fe})$ matte. Hydrometallurgy. 2006;51:332-337.

25 Teófilo F. Planejamentos experimentais para a otimização da resposta voltamétrica na determinação do herbicida glifosato em solo, água e vegetais [tese]. Viçosa: Programa de Pós-graduação em Agroquímica, Universidade Federal de Viçosa; 2003.

26 Lazić R. Design of experiments in chemical engineering. Catalonia: Wiley-VCH; 2004. . http://dx.doi. org/10.1002/3527604162.

27 Teófilo F, Ferreira C. Quimiometria II: planilhas eletrônicas para cálculos de planejamentos experimentais, um tutorial. Química Nova. 2006;29(2):338-350. http://dx.doi.org/10.1590/S0100-40422006000200026.

28 Cunico MWM, Cunico MM, Miguel OG, Zawadzki SF, Peralta-Zamora P, Volpato N. Planejamento fatorial: Uma ferramenta estatística valiosa para a definição de parâmetros experimentais empregados na pesquisa científica. Visão Acadêmica. 2008;9(1):23-32. http://dx.doi.org/10.5380/acd.v9i1.14635.

29 Bezerra A, Santelli E, Oliveira P, Villar S, Escaleira A. Response surface methodology (RSM) as a tool for optimization in analytical chemistry. Talanta. 2008;76(5):965-977. http://dx.doi.org/10.1016/j.talanta.2008.05.019.

30 Maia R. Método do vetor gradiente multivariado [dissertação]. Itajubá: Programa de Pós-graduação em Engenharia de Produção, Universidade Federal de Itajubá; 2013.

31 Pereira M, Gomes R, Rodrigues M, Leão V. Mechanical processing and characterization of printed circuit boards. In: Proceedings of European Metallurgical Conference; 2019 June 23-26; Düsseldorf, Germany. Clausthal-Zellerfeld: GDMB; 2019. p. 1019-1036.

32 Skoog D, West D, Holler F, Crouch R. Fundamentos de química analítica. 8. ed. São Paulo: Thomson; 2006.

33 Havlik T, Orac D, Petranikova M, Miskufova A, Kukurugya F, Takacova Z. Leaching of copper and tin from used printed circuit boards after thermal treatment. Journal of Hazardous Materials. 2010;183(1-3):866-873. http://dx.doi. org/10.1016/j.jhazmat.2010.07.107.

Recebido em: 30 Jan. 2019

Aceito em: 18 Mar. 2020 